\title{
On the investigation of some boundary value problems with non-linear conditions
}

\author{
Andrei Ronto and Miklós Rontó
}




\title{
ON THE INVESTIGATION OF SOME BOUNDARY VALUE PROBLEMS WITH NON-LINEAR CONDITIONS
}

\author{
AndRei Ronto \\ Institute of Mathematics, Ukrainian Academy of Sciences \\ 3 Tereshchenkovskaya Str., 01601 Kiev \\ ar@imath.kiev.ua \\ Miklós Rontó \\ Institute of Mathematics, University of Miskolc \\ 3515 Miskolc - Egyetemváros, Hungary \\ matronto@gold.uni-miskolc.hu
}

[Received July 22, 1999]

\begin{abstract}
A scheme of the numerical-analytic method based upon successive approximations is given for the investigation of solutions of nonlinear differential equations with nonlinear two-point boundary conditions.
\end{abstract}

Mathematical Subject Classification: 34B15

Keywords: Numerical-analytic-method, determining equations, nonlinear boundary-value problem, nonlinear boundary conditions

\section{Introduction}

The so-called numerical-analytic method of successive approximations $[1,2,3,4]$ is widely used for studying the solvability of non-linear boundary value problems and constructing approximate solutions. For a survey of applications and development of the method to problems of higher order ordinary differential and impulsive differential systems with affine (periodic, two- and multi-point) conditions, one can refer to the appropriate parts of the series of survey papers $[5,6]$

In $[7,3]$, the methodology of the numerical-analytic method is extended in order to make it possible to study the non-linear two-point boundary problem of the form

$$
\begin{gathered}
y^{\prime}(t)=f(t, y(t)), \quad t \in[0, T], \\
g(y(0), y(T))=0,
\end{gathered}
$$

for which purpose a general non-linear change of variable was introduced in Eq. (1.1). Here, we use a simpler substitution, which, as is shown, essentially facilitates the subsequent application of the numerical-analytic method. In particular, all the assumptions are formulated in terms of the original problem, and not the transformed one. 


\section{A reduction to the problem with linear conditions}

Consider the boundary value problem (1.1), (1.2), where the functions $f:[0, T] \times$ $G \rightarrow \mathbb{R}^{n}$ and $g: G \times G \rightarrow \mathbb{R}^{n}$ are continuous, $G \subset \mathbb{R}^{n}$ being the closure of a bounded domain. Assume that, for $t \in[0, T]$ fixed, $f$ satisfies the Lipschitz condition

$$
\left|f\left(t, y_{1}\right)-f\left(t, y_{2}\right)\right| \leq K\left|y_{1}-y_{2}\right|, \quad\left\{y_{1}, y_{2}\right\} \subset G,
$$

where the square matrix $K$ is supposed to have non-negative components. In (2.1), the absolute value sign and inequality are understood component-wise.

Let us introduce the traditional (see, e.g.,[8]) substitution

$$
y(t)=x(t)+w,
$$

where $w \in \Omega \subset \mathbb{R}^{n}$ is an unknown parameter. The domain, $\Omega$, of $w$ is chosen so that $D+\Omega \subset G$, whereas the new variable, $x$, is supposed to have range in $D$, the closure of a bounded subdomain of $G$.

Substitution (2.2) allows one to rewrite (1.1), (1.2) as

$$
\begin{gathered}
x^{\prime}(t)=f(t, x(t)+w), \quad t \in[0, T], \\
A x(0)+B x(T)=\phi(x(0)+w, x(T)+w)-[A+B] w,
\end{gathered}
$$

where $\phi(u, v):=A u+B v+g(u, v)$.

It is natural to determine the parameter $w$ from the additional equation

$$
\phi(x(0)+w, x(T)+w)-[A+B] w=0
$$

or, equivalently,

$$
A x(0)+B x(T)+g(x(0)+w, x(T)+w)=0 .
$$

Thus, the essentially non-linear problem (1.1), (1.2) turns out to be equivalent to problem (2.3), (2.4), and (2.5).

On the other hand, system $(2.3),(2.4),(2.5)$ can be regarded as a collection of problems

$$
\begin{gathered}
x^{\prime}(t)=f(t, x(t)+w), \\
A x(0)+B x(T)=0
\end{gathered}
$$

parametrised by $w$ belonging to a certain (unknown) set. The essential advantage obtained thereby is that the boundary condition (2.7) is linear. Therefore, problems of the family (2.6), (2.7) can be studied by using the numerical-analytic method developed in $[2,3]$.

Assume that

$$
D_{\beta}:=\left\{x \in \mathbb{R}^{n} \mid B(x, \beta(x)) \subset D\right\} \neq \emptyset,
$$


where

$$
\beta(x):=\frac{T}{4} \delta_{G}(f)+\left|\left(B^{-1} A+I_{n}\right) x\right|
$$

Here,

$$
\delta_{G}(f):=\max _{(t, y) \in[0, T] \times G} f(t, y)-\min _{(t, y) \in[0, T] \times G} f(t, y)
$$

Moreover, we suppose that

$$
r(K)<\frac{10}{3 T}
$$

Introduce the sequence of functions

$$
\begin{array}{rl}
x_{m+1}(t, w, z):=z+\int_{0}^{t} & f\left(s, x_{m}(s, w, z)+w\right) d s \\
& -\frac{t}{T} \int_{0}^{T} f\left(s, x_{m}(s, w, z)+w\right) d s-\frac{t}{T}\left[B^{-1} A+I_{n}\right] z,
\end{array}
$$

where $m=0,1, \ldots$ and $x_{0}(t, w, z) \equiv z$. It is easily seen that all the members of sequence (2.12) satisfy condition (2.7) for every $z \in D_{\beta}$ and $w \in \Omega$. Furthermore, $x_{m}(0, w, z)=z$ for all $m \in \mathbb{N}$.

By virtue of (2.7), a solution $x$ of (2.6), (2.7) satisfies

$$
x(T)=-B^{-1} A x(0)
$$

and, therefore, Eq. (2.5) can be rewritten as

$$
g\left(x(0)+w,-B^{-1} A x(0)+w\right)=0 .
$$

Summarising the above, we easily conclude that problem $(2.6),(2.7),(2.5)$ is equivalent to system $(2.6),(2.7),(2.13)$. We suggest solving it sequentially: first solve (2.6), (2.7), and then try to clarify whether (2.13) can be fulfilled.

Theorem 1 Assume conditions (2.1), (2.8), and (2.11). Then

1. Sequence $(2.12)$ has a limit $x^{*}(\cdot, w, z)$ uniform in $(t, w, z) \in[0, T] \times \Omega \times D_{\beta}$;

2. The limit function $x^{*}(\cdot, w, z)$ satisfies the 'perturbed' boundary value problem

$$
\begin{gathered}
x^{\prime}(t)=f(t, x(t)+w)+\Delta(w, z), \\
A x(0)+B x(T)=0,
\end{gathered}
$$

where

$$
\Delta(w, z):=-\frac{1}{T}\left[B^{-1} A+I_{n}\right] z-\frac{1}{T} \int_{0}^{T} f\left(s, x^{*}(s, w, z)+w\right) d s .
$$

Furthermore, $x^{*}(0, w, z)=z$. 
3. The error estimate

$$
\begin{array}{r}
\left|x^{*}(t, w, z)-x_{m}(t, w, z)\right| \leq \frac{20}{9} t\left(1-\frac{t}{T}\right) Q^{m-1}\left(I_{n}-Q\right)^{-1}\left[\frac{1}{2} Q \delta_{G}(f)\right. \\
\left.+K\left|\left(B^{-1} A+I_{n}\right) z\right|\right]
\end{array}
$$

holds, where $Q:=\frac{3 T}{10} K$ and $\delta_{G}(f)$ is as in (2.9).

Proof. It is carried out similarly to the proof of Theorem 1 from [9] and Theorem 2.1 from [3, p. 32].

The following statement shows the relation of the function $x^{*}(\cdot, w, z)$ to problem $(2.6),(2.7)$.

Theorem 2 Under assumptions (2.1), (2.8), and (2.11), the function $x^{*}\left(\cdot, w, z^{*}\right)$ is a solution of the boundary value problem (2.6), (2.7) if, and only if $z=z^{*}$ satisfies the 'determining equation',

$$
\left[B^{-1} A+I_{n}\right] z+\int_{0}^{T} f\left(s, x^{*}(s, w, z)+w\right) d s=0
$$

where $w$ is considered as a parameter.

Proof. Analogous to that of Theorem 2.4 from [3, p. 36].

Theorem 3 Assume conditions (2.1), (2.8), and (2.11). Then, for the function $x^{*}\left(\cdot, w, z^{*}\right)+w^{*}$ to be a solution of the boundary value problem (1.1), (1.2), it is sufficient that the parameters $z=z^{*}, w=w^{*}$ satisfy the system of determining equations

$$
\begin{gathered}
{\left[B^{-1} A+I_{n}\right] z+\int_{0}^{T} f\left(s, x^{*}(s, w, z)+w\right) d s=0} \\
g\left(z+w,-B^{-1} A z+w\right)=0
\end{gathered}
$$

In this case,

$$
y^{*}(t)=x^{*}\left(t, w^{*}, z^{*}\right)+w^{*}
$$

is a solution of the boundary value problem (1.1), (1.2).

Proof. It is obvious from the form of substitution (2.2) that equations (2.15) and (2.16) hold whenever the transformed boundary value problem (2.6), (2.7), (2.13) coincides with (1.1), (1.2). 
Remark 4 In [7,3], a change of variable more general than (2.2) was used,

$$
y(t)=x(t)+h(t, w)
$$

However, when $\partial h(t, w) / \partial t \not \equiv 0$, substitution of (2.18) leads to a more complicated transformed boundary value problem, as well as more complicated recursion sequence and determining equations. Furthermore, the basic assumptions should then be made on the transformed differential equation.

Remark 5 Considering (2.17), one can treat the function

$$
y_{m}(t)=x_{m}\left(t, w_{m}, z_{m}\right)+w_{m}
$$

as the mth approximation to the exact solution, $y^{*}\left(\cdot, w^{*}, z^{*}\right)$, of the boundary value problem (1.1), (1.2). In (2.19), $w_{m}$ and $z_{m}$ are solutions of the 'approximate determining equations',

$$
\begin{gathered}
{\left[B^{-1} A+I_{n}\right] z+\int_{0}^{T} f\left(s, x_{m}(s, w, z)+w\right) d s=0,} \\
g\left(z+w,-B^{-1} A z+w\right)=0
\end{gathered}
$$

and $x_{m}(\cdot, z, w)$ is given by (2.12). We do not consider the strict substantiation, referring the reader to [3] where similar techniques are described.

Remark 6 It follows from the consideration in [6] that the convergence of the recursion sequence $(2.12)$ can be proved under the condition $r(K)<3.4161 \ldots \cdot T^{-1}$, which is weaker than (2.11). However, estimate (2.14) does not hold in this case.

Remark 7 By using an idea from [4], one can obtain similar statements based on replacing (2.12) by the sequence

$$
\begin{aligned}
& x_{m+1}(t, w, z):=z-(A+B) z+\int_{0}^{t} f\left(s, x_{m}(s, w, z)+w\right) d s \\
&-\Phi(t) \int_{0}^{T} f\left(s, x_{m}(s, w, z)+w\right) d s,
\end{aligned}
$$

where $\Phi$ is an arbitrary continuous matrix-valued function such that

$$
A \Phi(0)+B \Phi(T)=I_{n}
$$

In this case, the restrictive conditions of type (2.11) can be avoided. However, an analogue of condition (2.8) is more difficult toverify unless $f$ in Eq. (1.1) is globally Lipschitzian.

Example 8 Consider the differential equation

$$
y^{\prime \prime}(t)+\frac{t}{16} y(t)+\frac{t}{4}\left(y^{\prime}(t)\right)^{2}=\frac{t^{2}}{128}, \quad t \in[0,1]
$$


under the non-linear boundary conditions

$$
\begin{aligned}
& y^{\prime}(1) y^{\prime}(0)+\frac{1}{4} y(0)=0 \\
& y(1) y^{\prime}(0)-\frac{1}{2} y^{\prime}(1)=-\frac{7}{128} .
\end{aligned}
$$

We reduce Eq. (2.22) to the first order system

$$
\begin{aligned}
& y_{1}^{\prime}(t)=y_{2}(t) \\
& y_{2}^{\prime}(t)=\frac{t^{2}}{128}-\frac{t}{16} y_{1}(t)-\frac{t}{4} y_{2}^{2}(t)
\end{aligned}
$$

conditions (2.23) then rewrite as

$$
\begin{aligned}
& y_{2}(1) y_{2}(0)+\frac{1}{4} y_{1}(0)=0 \\
& y_{1}(1) y_{2}(0)-\frac{1}{2} y_{2}(1)=-\frac{7}{128} .
\end{aligned}
$$

Put $A=B=I_{2}$ and $T=1$. It is not difficult to verify that the assumptions of the theorems above hold in the domain $G:=\left\{\left(y_{1}, y_{2}\right)|| y_{1}|\leq 1,| y_{2} \mid \leq \frac{1}{2}\right\}$.

Namely, (2.8) holds when $\beta$ from (2.9) satisfies the component-wise inequality $\beta\left(x_{1}, x_{2}\right) \leq\left(\begin{array}{c}\frac{1}{4} \\ \frac{17}{256}\end{array}\right)+2\left(\begin{array}{l}x_{1} \\ x_{2}\end{array}\right)$. The vector function in the right-hand side of $(2.24)$ satisfies (2.1) in $G$ with $K=\left(\begin{array}{cc}0 & 1 \\ \frac{1}{16} & \frac{1}{4}\end{array}\right)$. Since $r(K)=\frac{1+\sqrt{2}}{4}$, it is obvious that (2.11) holds.

Substitution (2.2) in (2.24) leads us to the system

$$
\begin{aligned}
x_{1}^{\prime}(t) & =x_{2}(t)+w_{2}, \\
x_{2}^{\prime}(t) & =\frac{t^{2}}{128}-\frac{t}{16}\left[x_{1}(t)+w_{1}\right]-\frac{t}{4}\left[x_{2}(t)+w_{2}\right]^{2} .
\end{aligned}
$$

In this case, the determining equation (2.21) has the form

$$
\begin{aligned}
w_{2}^{2}-z_{2}^{2}+\frac{1}{4}\left(z_{1}+w_{1}\right) & =0 \\
\left(w_{1}-z_{1}\right)\left(w_{2}+z_{2}\right)-\frac{1}{2}\left(w_{2}-z_{2}\right) & =-\frac{7}{128} .
\end{aligned}
$$

Let us find the first approximation, $x_{1}=\left(\begin{array}{l}x_{1,1} \\ x_{1,2}\end{array}\right)$, in the sense of Remark 5. According to (2.12), we have

$$
\begin{aligned}
x_{1,1}\left(t, w_{1}, w_{2}, z_{1}, z_{2}\right) & =z_{1}-2 t z_{1} \\
x_{1,2}\left(t, w_{1}, w_{2}, z_{1}, z_{2}\right) & =z_{2}+\frac{t^{3}}{384}-\frac{t^{2}}{32}\left(z_{1}+w_{1}\right)-\frac{t^{2}}{8}\left(z_{1}+w_{2}\right)^{2}-\frac{t}{384} \\
& +\frac{t}{32}\left(z_{1}+w_{1}\right)+\frac{t}{8}\left(z_{2}+w_{2}\right)^{2}-2 t z_{2} .
\end{aligned}
$$


The first approximate determining equation (2.20) (i.e., that corresponding to $m=$ $1)$, is equivalent to $\Delta_{1}(w, z)=0$, where $\Delta_{1}=\left(\begin{array}{c}\Delta_{1,1} \\ \Delta_{1,2}\end{array}\right)$ is given by

$$
\begin{aligned}
\Delta_{1,1}\left(w_{1}, w_{2}, z_{1}, z_{2}\right) & =\frac{385}{192} z_{1}-\frac{1}{1536}+\frac{1}{192} w_{1} \\
& +\frac{1}{48}\left(z_{2}+w_{2}\right)^{2}+w_{2}
\end{aligned}
$$

and

$$
\begin{aligned}
\Delta_{1,2}\left(w_{1}, w_{2}, z_{1}, z_{2}\right)=-\frac{1}{15360} z_{1} z_{2} w_{2}+\frac{36863}{14155776}+\frac{107531}{10321920} z_{1} \\
+\frac{46079}{23040} z_{2}-\frac{322549}{10321920} w_{1}+\frac{1}{5760} w_{2}-\frac{1}{15360} w_{1} z_{2} w_{2} \\
-\frac{1}{15360} z_{2}{ }^{4}-\frac{1}{15360} w_{2}{ }^{4}-\frac{107509}{2580480} z_{2}{ }^{2}+\frac{107531}{1290240} z_{2} w_{2} \\
-\frac{322549}{2580480} w_{2}{ }^{2}-\frac{1}{245760} z_{1}{ }^{2}-\frac{1}{122880} z_{1} w_{1}-\frac{1}{30720} z_{1} z_{2}{ }^{2} \\
-\frac{1}{30720} z_{1} w_{2}{ }^{2}-\frac{1}{245760} w_{1}^{2}-\frac{1}{30720} w_{1} z_{2}{ }^{2}-\frac{1}{30720} w_{1} w_{2}{ }^{2} \\
-\frac{1}{3840} z_{2}{ }^{3} w_{2}-\frac{1}{2560} z_{2}{ }^{2} w_{2}{ }^{2}-\frac{1}{3840} z_{2} w_{2}{ }^{3}+\frac{1}{3840} z_{2} z_{1} \\
\quad+\frac{1}{3840} z_{2} w_{1}-\frac{1}{320} z_{2}{ }^{2} w_{2}-\frac{3}{320} z_{2} w_{2}{ }^{2}-\frac{1}{768} w_{2} z_{1} \\
-\frac{1}{768} w_{2} w_{1}+\frac{1}{960} z_{2}{ }^{3}-\frac{1}{192} w_{2}^{3} .
\end{aligned}
$$

Solving system (2.25) together with the approximate determining equations

$$
\Delta_{1,1}\left(w_{1}, w_{2}, z_{1}, z_{2}\right)=0, \quad \Delta_{1,2}\left(w_{1}, w_{2}, z_{1}, z_{2}\right)=0,
$$

where $\Delta_{1,1}$ and $\Delta_{1,2}$ are given by (2.26) and (2.27), we obtain the following values for $z_{1}=\left(\begin{array}{l}z_{1,1} \\ z_{1,2}\end{array}\right)$ and $w_{1}=\left(\begin{array}{l}w_{1,1} \\ w_{1,2}\end{array}\right)$ :

$$
\begin{aligned}
z_{1,1} & \approx-0.06206890391, & z_{1,2} & \approx-0.00001967788609, \\
w_{1,1} & \approx-0.0002202244703, & w_{1,2} & \approx 0.1247889518 .
\end{aligned}
$$

With these values of parameters, the first approximation, $x_{1}$, has the form

$$
\begin{aligned}
x_{1,1}(t) & \approx-0.06228912838+0.1241378078 t \\
x_{1,2}(t) & \approx-0.196778861 \cdot 10^{-4}+\frac{1}{384} t^{3}+0.613798 \cdot 10^{-6} t^{2} \\
& -0.2565424693 \cdot 10^{-2} t .
\end{aligned}
$$

Consequently, according to substitution (2.2), the solution, y, of problem (2.22), (2.23), up to the first approximation $y_{1}(t)=x_{1}(t)+w_{1}$, has the form 


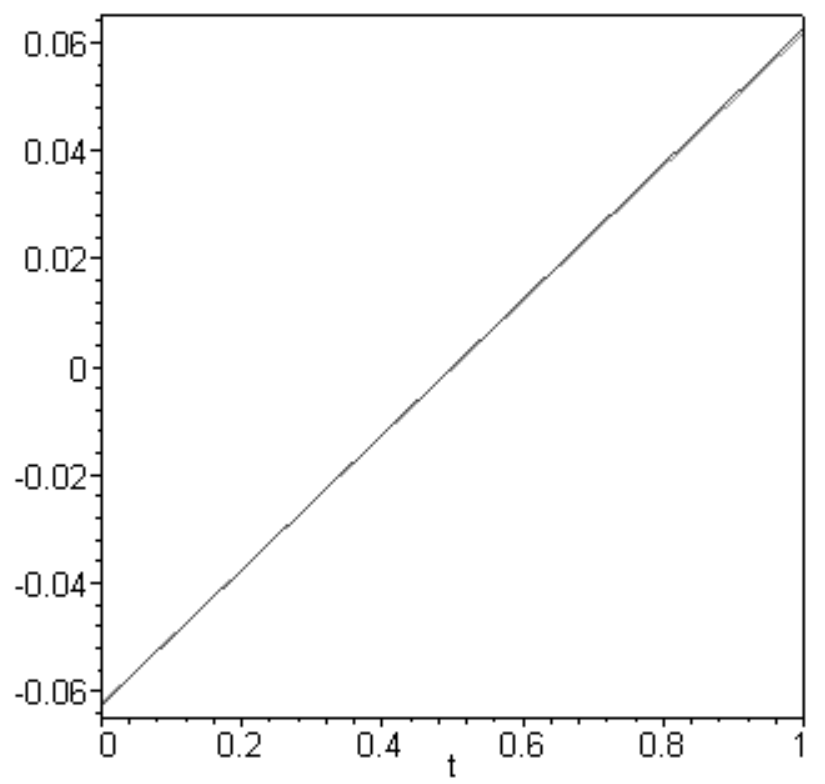

Figure 1.

$$
\begin{aligned}
y_{1,1}(t) & \approx-0.06250935285+0.1241378078 t \\
y_{1,2}(t) & \approx 0.1247692739+\frac{1}{384} t^{3}+0.613798 \cdot 10^{-6} t^{2} \\
& -0.2565424693 \cdot 10^{-2} t .
\end{aligned}
$$

Note that the function $y^{*}(t)=\frac{t}{8}-\frac{1}{16}$ is an exact solution of problem (2.22), (2.23). As is seen from Figures 1 and 2, the graphs of the exact solution and the first approximation almost coincide, whereas the deviation of their derivatives does not exceed 0.001. Thus, even the first iteration of the method gives a satisfactory approximation for the solution of the problem considered.

\section{Separated non-linear boundary conditions}

It turns out that, in the case of separated, in a sense, boundary conditions, the numerical-analytic method can be applied without any change of variable.

Consider the two-point boundary value problem

$$
\begin{aligned}
& x^{\prime}(t)=f(t, x(t)), \quad t \in[0, T], \\
& x(T)=a(x(0)),
\end{aligned}
$$




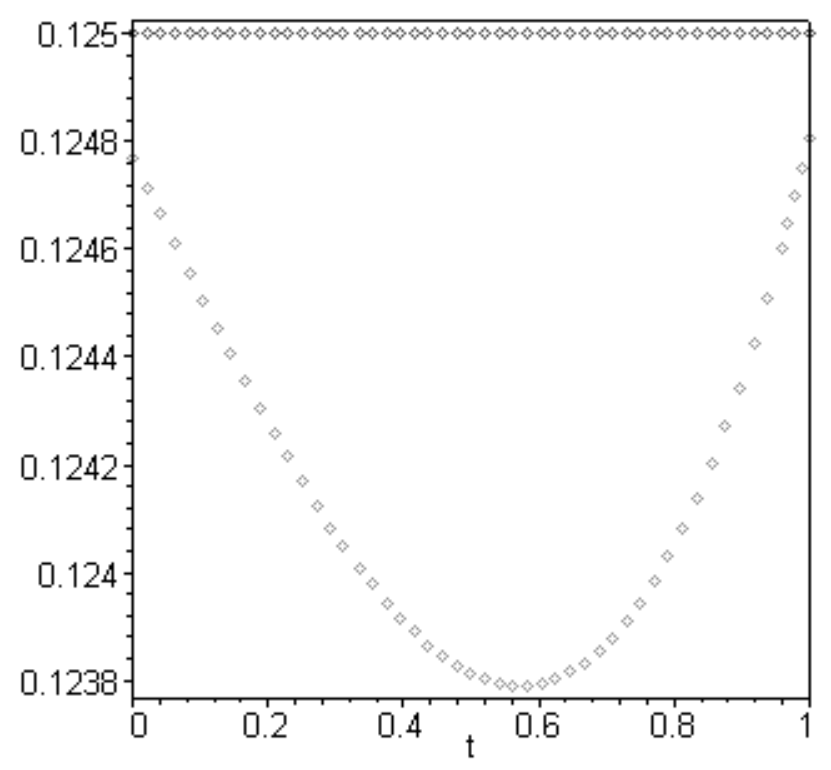

Figure 2.

where $a: D \rightarrow \mathbb{R}^{n}$ is continuous and $f$ satisfies conditions (2.1) and (2.11) in the closure, $D$, of a bounded domain from $\mathbb{R}^{n}$.

Put $\gamma(x):=\frac{T}{4} \delta_{D}(f)+|a(x)-x|$ and assume that

$$
D_{\gamma} \neq \emptyset
$$

where the set $D_{\gamma}$ is defined similarly to $(2.8)$, whereas the number $\delta_{D}(f)$ is given by (2.10).

Introduce the sequence of functions

$$
\begin{aligned}
x_{m+1}(t, z):=z+\int_{0}^{t} f\left(s, x_{m}(s, z)\right) d s & \\
& -\frac{t}{T} \int_{0}^{T} f\left(s, x_{m}(s, z)\right) d s+\frac{t}{T}[a(z)-z],
\end{aligned}
$$

where $m=0,1, \ldots$ and $x_{0}(t, z) \equiv z \in D_{\gamma}$. Obviously, all the members of sequence (3.4) satisfy condition (3.1) for every $z \in D_{\gamma}$.

Theorem 9 Assume conditions (2.1), (2.11), and (3.3). Then: 
1. Sequence (3.4) converges uniformly in $[0, T] \times D_{\gamma}$,

$$
\lim _{n \rightarrow+\infty} \sup _{(t, z) \in[0, T] \times D_{\gamma}}\left\|x_{n}(t, z)-x^{*}(t, z)\right\|=0 .
$$

2. The function $x^{*}(\cdot, z)$ is a solution of the 'modified' problem,

$$
\begin{aligned}
& x^{\prime}(t)=f(t, x(t))+\Delta(z), \quad t \in[0, T], \\
& x(T)=a(x(0)),
\end{aligned}
$$

where

$$
T \Delta(z):=a(z)-z-\int_{0}^{T} f\left(s, x^{*}(s, z)\right) d s .
$$

Moreover, $x^{*}(\cdot, z)$ satisfies $x^{*}(0, z)=x_{m}(0, z)=z(\forall m \in \mathbb{N})$.

3. The error estimate

$$
\begin{array}{r}
\left|x^{*}(t, w, z)-x_{m}(t, w, z)\right| \leq \frac{20}{9} t\left(1-\frac{t}{T}\right) Q^{m-1}\left(I_{n}-Q\right)^{-1}\left[\frac{1}{2} Q \delta_{D}(f)\right. \\
+K|a(z)-z|]
\end{array}
$$

holds, where $Q:=\frac{3 T}{10} K$ and $\delta_{D}(f)$ is defined by $(2.10)$.

Proof. Analogous to that of Theorem 2.1 from [3, p. 32].

Theorem 10 Under conditions of Theorem 9, the limit function of sequence (3.4) is a solution of problem (3.1), (3.2) if, and only if the parameter $z=z^{*}$ (which stands for the initial value of the solution at $t=0$ ) satisfies the 'determining equation,'

$$
a(z)-z=\int_{0}^{T} f\left(s, x^{*}(s, z)\right) d s .
$$

Proof. Similar to that of Theorem 2.3 from [3, p. 36].

Remark 11 When, in (3.1), a(z) 三z, then (3.1), (3.2) is nothing but the T-periodic boundary value problem. In this case, sequence (3.4) is reduced to the well-known [1] T-periodic successive approximations,'

$$
x_{m+1}(t, z):=z+\int_{0}^{t} f\left(s, x_{m}(s, z)\right) d s-\frac{t}{T} \int_{0}^{T} f\left(s, x_{m}(s, z)\right) d s .
$$

Remark 12 Estimate (3.5) implies that the function $t \mapsto x_{m}\left(t, z_{m}\right)$ is natural to be taken as the mth approximation of the exact solution of problem (3.1), (3.2), when $z=z_{m}$ is a root of the 'mth approximate determining equation,'

$$
a(z)-z=\int_{0}^{T} f\left(s, x_{m}(s, z)\right) d s .
$$


Example 13 Let us apply the technique described above to the problem

$$
\begin{aligned}
& x^{\prime \prime}(t)-\frac{t}{8} x^{\prime}(t)+\frac{1}{2}\left(x^{\prime}(t)\right)^{2}=\frac{1}{4}, \quad t \in[0,1], \\
& x(1)=\frac{1}{2}\left(x^{\prime}(0)\right)^{2}+\frac{1}{16}, \\
& x^{\prime}(1)=-x(0)+\frac{3}{16} .
\end{aligned}
$$

As usual, we rewrite the second order problem (3.7) in the form

$$
\begin{aligned}
x_{1}^{\prime}(t) & =x_{2}, \\
x_{2}^{\prime}(t) & =\frac{1}{4}-\frac{t}{8} x_{2}(t)-\frac{1}{2} x_{2}^{2}(t), \quad t \in[0,1], \\
x_{1}(1) & =\frac{1}{2} x_{2}^{2}(0)+\frac{1}{16} \\
x_{2}(1) & =-x_{1}(0)+\frac{3}{16} .
\end{aligned}
$$

Consider (3.8) in the domain

$$
\left(t, x_{1}, x_{2}\right) \in[0,1] \times D, \quad D=\left\{\left(x_{1}, x_{2}\right)|| x_{1}|\leq 1,| x_{2} \mid \leq \frac{3}{4}\right\} .
$$

It is not difficult to verify that the conditions of Theorem 9 are fulfilled with $K=\left(\begin{array}{ll}0 & 1 \\ 0 & \frac{7}{8}\end{array}\right), \gamma(x) \leq\left(\begin{array}{c}\frac{7}{16}+\frac{1}{2} x_{2}^{2}-x_{1} \\ \frac{1}{2}-x_{1}-x_{2}\end{array}.\right)$ The corresponding quantity (2.10) is estimated as $\delta_{D}(f) \leq\left(\begin{array}{c}\frac{3}{4} \\ \frac{5}{8}\end{array}\right)$, and $K$ satisfies $(2.11)$ because $r(K)=\frac{7}{8}$.

According to (3.4), we have the following formula for $x_{1}=\left(\begin{array}{l}x_{1,1} \\ x_{1,2}\end{array}\right)$ :

$$
\begin{aligned}
& x_{1,1}\left(t, \zeta_{1}, \zeta_{2}\right)=\zeta_{1}+t\left(\frac{1}{2} \zeta_{2}^{2}-\zeta_{1}+\frac{1}{16}\right) \\
& x_{1,2}\left(t, \zeta_{1}, \zeta_{2}\right)=\zeta_{2}+\frac{t^{2}}{16} \zeta_{2}-\frac{1}{16} t \zeta_{2}-t\left(\zeta_{1}+\zeta_{2}-\frac{3}{16}\right) .
\end{aligned}
$$

The first approximate determining equations (3.6) has the form

$$
\Delta_{1,1}\left(\zeta_{1}, \zeta_{2}\right)=0, \Delta_{1,2}\left(\zeta_{1}, \zeta_{2}\right)=0
$$

where

$$
\begin{aligned}
\Delta_{1,1}\left(\zeta_{1}, \zeta_{2}\right) & =\frac{1}{2} \zeta_{2}^{2}-\frac{47}{96} \zeta_{2}-\frac{1}{2} \zeta_{1}-\frac{1}{32} \\
\Delta_{1,2}\left(\zeta_{1}, \zeta_{2}\right) & =-\frac{49}{48} \zeta_{1}-\frac{33}{512}-\frac{88165}{89088} \zeta_{2}+\frac{827}{5120} \zeta_{2}{ }^{2}-\frac{31}{192} \zeta_{2} \zeta_{1}+\frac{1}{6} \zeta_{1}{ }^{2}
\end{aligned}
$$

The solution, $\zeta=\left(\begin{array}{l}\zeta_{1} \\ \zeta_{2}\end{array}\right)$, of system (3.12) has the form

$$
\zeta_{1}=-\frac{1}{16}, \quad \zeta_{2}=0
$$


Inserting (3.13) into (3.10) and (3.11), we obtain the first approximation

$$
x_{1,1}(t)=\frac{t}{8}-\frac{1}{16}, \quad x_{1,2}(t)=\frac{t}{4} .
$$

Note that the vector function with components

$$
x_{1}^{*}(t)=\frac{t^{2}}{8}-\frac{1}{16}, \quad x_{2}^{*}(t)=\frac{t}{4}
$$

is an exact solution of problem (3.8).

Let us construct the second approximation. By (3.4), we have

$$
\begin{aligned}
x_{2,1}\left(t, \zeta_{1}, \zeta_{2}\right) & =\zeta_{1}+\frac{1}{48} \zeta_{2} t^{3}-\frac{1}{2} t^{2} \zeta_{1}+\frac{3}{32} t^{2}-\frac{17}{32} t^{2} \zeta_{2} \\
& +\frac{49}{96} \zeta_{2} t-\frac{1}{2} t \zeta_{1}-\frac{1}{32} t+\frac{1}{2} t \zeta_{2}^{2}, \\
x_{2,2}\left(t, \zeta_{1}, \zeta_{2}\right) & =\frac{95}{512} t-\frac{31}{192} t \zeta_{2} \zeta_{1}+z_{2}+\frac{17}{768} \zeta_{2} t^{3}-\frac{1}{32} t^{2} \zeta_{2}-\frac{49}{48} t \zeta_{1} \\
& -\frac{1733}{5120} t \zeta_{2}^{2}+\frac{1}{512} t^{3}-\frac{3041}{3072} \zeta_{2} t-\frac{1}{2560} \zeta_{2}^{2} t^{5}+\frac{1}{64} t^{4} \zeta_{2} \zeta_{1} \\
& -\frac{17}{48} t^{3} \zeta_{2} \zeta_{1}+\frac{1}{2} t^{2} \zeta_{2} \zeta_{1}-\frac{1}{1024} t^{4} \zeta_{2}+\frac{17}{1024} t^{4} \zeta_{2}^{2}+\frac{1}{48} t^{3} \zeta_{1} \\
& -\frac{107}{512} t^{3} \zeta_{2}^{2}-\frac{1}{6} t^{3} \zeta_{1}^{2}+\frac{17}{32} t^{2} \zeta_{2}^{2}+\frac{1}{6} t \zeta_{1}^{2} .
\end{aligned}
$$

It is not difficult to verify that inserting into (3.15) and (3.16) even the roots (3.13) of the first determining system (3.12), we arrive at the exact solution (3.14) of problem (3.8).

Acknowledgement: The first author was supported in part by the Hungarian-Ukrainian Bilateral Science \& Technology Co-operation Programme through grant No. UK-3/99. The second author was supported in part by the Hungarian National Research Foundation through grant No. T031961.

\section{REFERENCES}

[1] Samollenko, A. M. and Ronto, N. I.: Numerical-Analytic Methods of Investigating Periodic Solutions, Mir, Moscow, 1980.

[2] Samollenko, A. M. and Ronto, N. I.: Numerical-Analytic Methods of Investigating Solutions of Boundary Value Problems, Naukova Dumka, Kiev, 1985. (in Russian)

[3] Samollenko, A. M. and Ronto, N. I.: Numerical-Analytic Methods in the Theory of Boundary Value Problems for Ordinary Differential Equations, Naukova Dumka, Kiev, 1992. (in Russian)

[4] Ronto, A.: On the boundary value problems with linear multipoint restrictions, Publ. Univ. of Miskolc, Ser. D. Natur. Sc. Math., 36(1), (1995), 81-89.

[5] Samoilenko, A. M., Ronto, N. I. and Trofimchuk, S. I.: Theory of numericalanalytic method. Achievements and new trends of development. I, Ukr. Mat. Zh., 50(1), (1998), 102-117. 
[6] Samollenko, A. M., Ronto, N. I. and Trofimchuk, S. I.: Theory of numericalanalytic method. Achievements and new trends of development. VI, Ukr. Mat. Zh., 51(7), (1999), 960-971.

[7] Samollenko, A. M. and Le Lyuong TAi: On a method of investigating boundary value problems with non-linear boundary conditions, Ukr. Mat. Zh., 42(7), (1990), 951-957.

[8] Bogolyubov, N. N. and Mitropol'skit,Yu. A.: Asymptotic Methods in the Theory of Non-Linear Oscillations, 2nd rev. and aug. ed., Fizmatgiz, Moscow, 1958. (in Russian)

[9] Rontó, M.: On numerical-analytic method for BVPs with parameters, Publ. Univ. of Miskolc, Ser. D. Natur. Sc. Math., 36(2), (1996), 125-132. 